J. Clin. Chem. Clin. Biochem.

Vol. 14, 1976, pp. 333-337

\title{
Die Bestimmung der Cystinaminopeptidase (Oxytocinase) mit einem ENI-Fast-Analyzer ${ }^{1}$ )
}

\author{
Von H. Wisser, K. Dettmer und E. Knoll \\ Abteilung für Klinische Chemie, Robert-Bosch-Krankenhaus, Stuttgart
}

Zusammenfassung: Ein Verfahren zur Bestimmung der Cystinaminopeptidase-Aktivität wurde auf einen Fast-Analyzer adaptiert. Die verschiedenen Reaktionsparameter wurden überprüft. Die Präzision des Verfahrens liegt bei etwa $5 \%$. Die Cystinaminopeptidase-Aktivität bleibt auch bei längerer Aufbewahrung bei $+4^{\circ} \mathrm{C}$ und $-20^{\circ} \mathrm{C}$ stabil. An einer Stichprobe von Patientinnen mit normalem Schwangerschaftsverlauf wurde der Normbereich für die letzten Schwangerschaftswochen bestimmt.

\section{The determination of cystine aminopeptidase (oxytocinase) with a ENI fast analyzer}

Summary: A procedure for the determination of cystine aminopeptidase activity was adapted to a fast analyzer. The various reaction parameters were checked. The precision of the method was about $5 \%$. Cystine aminopeptidase activity was stable for long storage periods at $+4^{\circ} \mathrm{C}$ and $-20^{\circ} \mathrm{C}$. The normal range for the terminal weeks of pregnancy was determined on samples from female patients with normal pregnancies.

\section{Einleitung}

Die Herkunft der aus mehreren Isoenzymen bestehenden Cystinaminopeptidase (EC 3.4.11.3) aus der Placenta konnte durch histochemische und immunhistochemische Verfahren nachgewiesen werden. In der Schwangerschaft steigt die Cystinaminopeptidase-Aktivität des Serums an. Uber die Bedeutung ihrer Bestimmung als Parameter zur Erkennung einer Störung der foetoplacentaren Einheit liegen bisher widersprüchliche Angaben vor (Ubersicht s. 1. c. (1)). Die Cystinaminopeptidase-Aktivität wurde mit verschiedenen Substraten, Naphthyl- und $p$-Nitrophenylamiden des Cysteins bzw. Cystins, sowohl kinetisch als auch durch Endpunktbestimmung ermittelt (2-9). In Anlehnung an die Untersuchungen von Oudheusden $(4,5)$ und Tovey et al. (7) wurde das Verfahren auf den ENI-Fast-Analyzer ${ }^{2}$ ) adaptiert, mit dem Ziel, ein einfaches, schnelles Verfahren guter Präzision zur Bestimmung der Cystinaminopeptidase-Aktivität zu entwickeln. Die Bestimmung soll in einer Studie über die Wertigkeit verschiedener Parameter zur Erkennung einer Störung der foetoplacentaren Einheit eingesetzt werden. Die Verwendung von S-Benzyl- $L$-cystein-p-nitroanilid als Substrat hat den Vorteil, daß es sehr schnell gespalten wird.

\footnotetext{
1) Diese Arbeit wurde unterstützt aus Mitteln der Robert-BoschStiftung, Stuttgart.
}

\section{Methodik}

Die Cystinaminopeptidase (Oxytocinase) katalysiert die Hydrolyse des S-Benzyl- $L$-cystein- $p$-nitroanilids. Die nach einer bestimmten Zeit freigesetzte $p$-Nitroanilinmenge ist proportional der Cystinaminopeptidase-Aktivität und kann durch Messung der Absorption des p-Nitroanilins bei $405 \mathrm{~nm}$ gemessen werden.

Geräte

Fast-Analyzer mit Rotoloader ${ }^{2}$ )

Reagenzien und Lösungen

1. Substratlösung $(7,55 \mathrm{mmol} / \mathrm{l})$ $25 \mathrm{mg}$ S-Benzyl- $L$-cystein-p-nitroanilid ${ }^{3}$ ) werden in $10 \mathrm{ml}$ Methoxyethanol (Ethylenglykolmonomethyläther) (Merck Nr. 859) gelöst. In dunkler Flasche bei $4-6^{\circ} \mathrm{C}$ aufbewahrt etwa einen Monat haltbar.

2. Phosphatpuffer $0,1 \mathrm{~mol} / 1, \mathrm{pH} 7,3$

$13,61 \mathrm{~g} \mathrm{KH}_{2} \mathrm{PO}_{4}$ (Merck Nr. 4873) werden in etwa $900 \mathrm{ml}$ bidest. Wasser gelöst, mit $1 \mathrm{~mol} / 1$ Natronlauge auf $\mathrm{pH} 7,3$ eingestellt und auf 1 Liter aufgefüllt.

3. 0,15 mol/1 NaCl-Lösung

\section{Arbeitsweise}

$500 \mu 1$ Phosphatpuffer, $50 \mu$ l Probe und $100 \mu l$ Natriumchloridlösung (als Spülflüssigkeit) werden mit dem Rotoloader pipettiert. $50 \mu \mathrm{l}$ Substratlösung werden mit einer Eppendorf-Pipette pipettiert, da die normalen Zufuhrschläuche der Pumpen durch

2) Firma Electro Nucleonics, Stuttgart.

3) Herrn Dr. Lang, Biochemische Forschung der Firma Merck, danken wir für die Uberlassung einer größeren Menge dieser Substanz. 
den Ethylenglykolmonomethyläther angegriffen werden. In Position 1 wird Wasser, in die Positionen 2 und 3 Kontrollen und in die Positionen 4-16 Patientenproben pipettiert.

\section{Pipettierung \\ $500 \mu \mathrm{l}$ Puffer in Position C \\ $50 \mu \mathrm{l}$ Probe in Position C \\ $100 \mu \mathrm{l} \mathrm{NaCl}-L o ̈ s u n g$ in Position C \\ $50 \mu$ l Substrat in Position B mit Eppendorf-Pipetten}

Meßbedingungen
Filter $405 \mathrm{~nm}$ (Filter-Range $385-430 \mathrm{~nm}$ )
$\begin{array}{lc}\text { Erste Messung } & 120 \mathrm{~s} \\ \text { Meßintervall: } & 60 \mathrm{~s} \\ \text { Anzahl der Messungen: } & 4 \\ \text { Umrechnungsfaktor } & 1414 \\ \text { Meßtemperatur } & 25^{\circ} \mathrm{C}\end{array}$

Ergebnisse und Diskussion

Verschiedene Parameter, die von Einfluß auf die Bestimmung sind, wurden variiert. Über die Ergebnisse wird im folgenden berichtet.

\section{Methoxyethanolkonzentration}

Von verschiedenen Untersuchern $(3,4,5,7)$ wurde zur Erhöhung der Löslichkeit des Substrates dem Reagenz: gemisch Methoxyethanol zugesetzt. Neben der Löslichkeitserhöhung wurde eine Aktivitätssteigerung beobachtet, allerdings nur in einem gewissen Konzentrationsbereich. In Übereinstimmung mit den Ergebnissen von Tovey et al. (7) fanden wir bei Verwendung des gleichen Substrates ebenfalls die größte Aktivitätssteigerung bei einer Endkonzentration des Methoxyethanols von 80-100 ml/l und über $120 \mathrm{ml} / \mathrm{l}$ einen Aktivitätsabfall.

\section{2. pH-Optimum und Konzentration des Phosphatpuffers}

Die Überprüfung der pH-Abhängigkeit der Cystinaminopeptidase-Aktivität ergab ein pH-Optimum zwischen pH 7,2-7,5 des Phosphatpuffers. Dies stimmt mit den Ergebnissen anderer Untersucher $(5,7)$ überein. Neben dem pH-Wert wurde der Einfluß der Konzentration des Puffers untersucht. Dabei ist bis zu einer Konzentration von $0,1 \mathrm{mmol} / \mathrm{l}(\mathrm{pH} 7,3)$ die Aktivität unverändert. Bei höheren Konzentrationen nimmt die Aktivität ab (Abb. 1).

\section{Substratkonzentration}

Die Ermittlung der optimalen Substratkonzentration ist insofern schwierig, da es bei einer Substratkonzentration zwischen 0,54 und 0,94 mmol/1 Ansatz trotz Lösungsvermittlerzusatz zu einer Ausfällung des Substrats kommt. Die Abhängigkeit der Aktivität von der steigenden Substratkonzentration ist in folgender Abbildung 2 wiedergegeben.

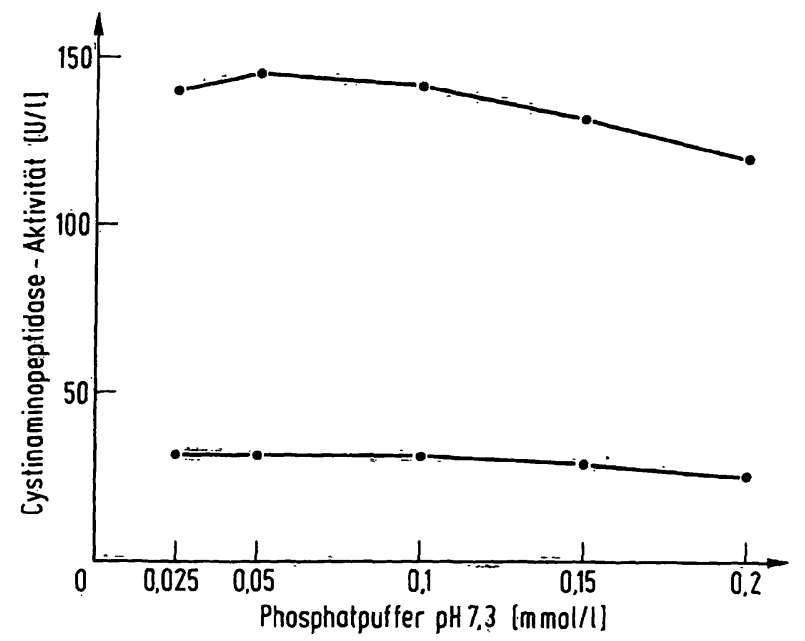

Abb. 1. Einfluß der Konzentration des P̈hosphatpuffers auf die Cystinaminopeptidase-Aktivität.

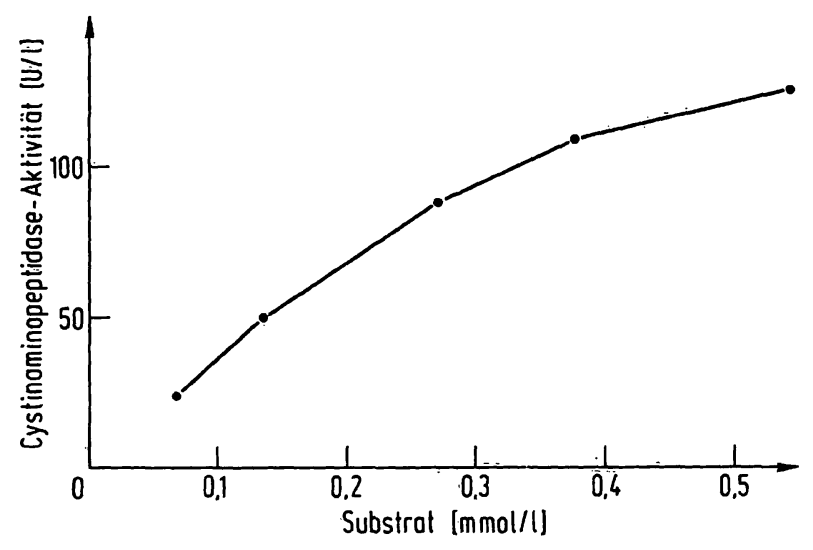

Abb. 2. Abhängigkeit der Cystinaminopeptidase-Aktivität von der Substratkonz̈entration im Ansatz.

\section{Linearität}

Es wurde geprüft; ob eine lineare Abhängigkeit von Aktivität und Enzymkonzentration besteht. Dazu wurde ein Schwangerenserum mit hoher CystinaminopeptidaseAktivität mit Nichtschwangerenserum unterschiedlich verdünnt und in den Verdünnungen die Cystinaminopeptidạse-Aktivität bestimmt. Wie die Abbildung 3 zeigt, besteht für den untersuchten Konzentrationsbereich eine lineare Abhängigkeit von Aktivität und' Konzentration.

In einer jüngst erschienenen Publikation (10) wird auf Störungen beim Bestimmungsverfahren mit dem „Ênzyrator" hingewiesen. Diese bestanden in einer Abnahme der Aktivität während der ersten 2-3 Minuten, dann einer konstanten Aktivität und schließlich einer erneutcn Abnahme. Das Ausmaß dieser Störung war abhängig "on der Enzymaktivität.

Diese Befunde können wir nach unșeren Ergebnissen nur zum Teil und auch nicht in dem Ausmaß, wie sie in Abbildung 1 der erwähnten Publikation (10) wiedergegeben sind, beștätigen. Vier Seren unterschiedlicher Aktivität, 


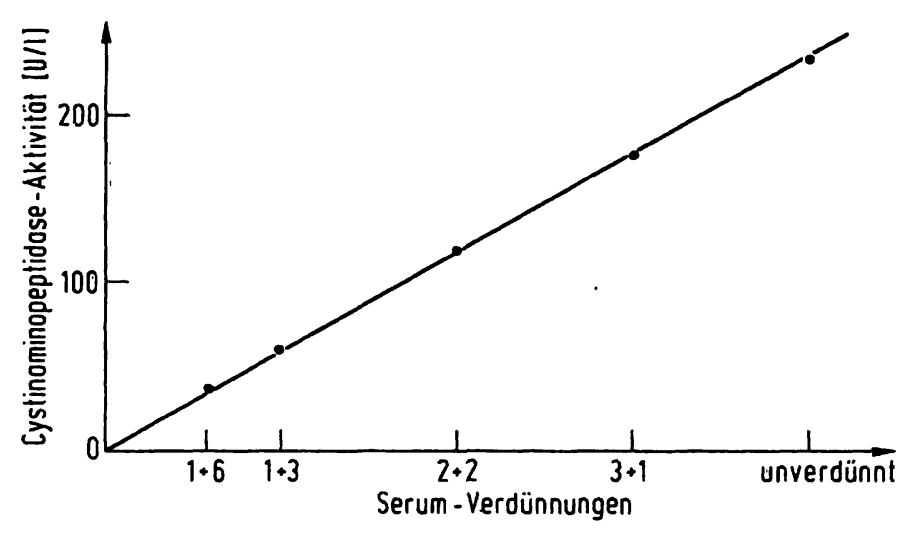

Abb. 3. Cystinaminopeptidase-Aktivität bei steigender Verdünnung des gleichen Serums mit Nichtschwangerenserum.

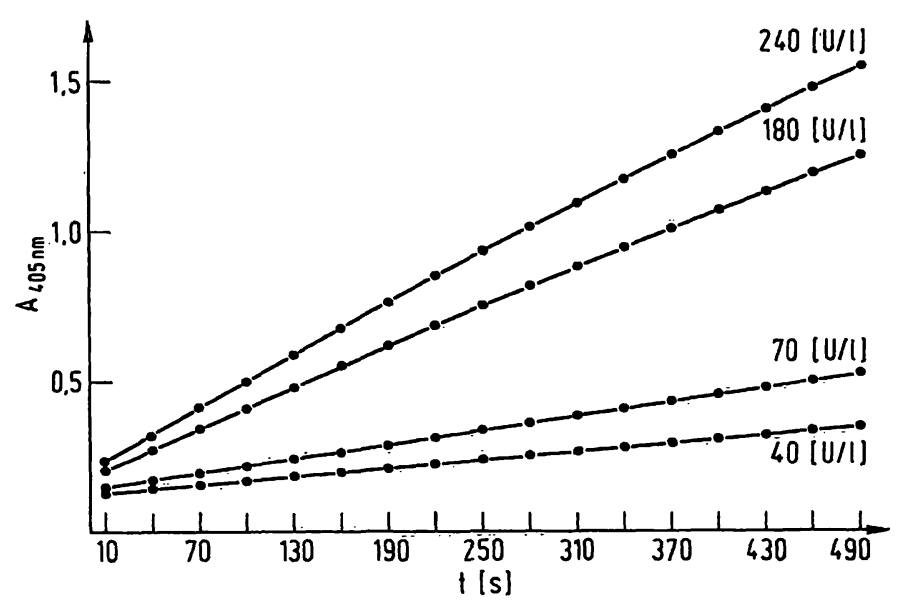

Aọb. 4. Zeitverlauf der Absorptionszunahme bei Analyse von 4 Seren unterschiedlicher Aktivität.

die ron uns untersucht wurden, zeigen bis zu einer Reaktionszeit von $250 \mathrm{~s}$ eine lineare Abhängigkeit von Absorption und Konzentration (Abb. 4). Bei den Seren mit den beiden höchsten Enzymaktivitäten mächt sich nach dieșer Zeit eine leichte Krümmung bemerkbar. Diès könnte durch einen Hemmeffekt des freigesetzten $p$ Nitroanilins bedingt sein (10). Man wird diesen Effekt bei Aktivitäten von $180 \mathrm{U} / 1$ und mehr berücksichtigen müssen, indem man das Meßintervall von $60 \mathrm{~s}$ auf $30 \mathrm{~s}$ verkürūzt.

\section{Stabilität des Enzymes}

Zur Frage der Konstanz der CystinaminopeptidaseAktivität war es erforderlich, zu prüfen, wie diese sich unter verschiedenen Versuchsbedingungen verhält. Dazu wưryèn 10 verschiedene Seren von Schwangeren über einen Żeitraum von 21 Tagen bei Aufbewahrung der Proben bei $+4^{\circ} \mathrm{C}$ und $-20^{\circ} \mathrm{C}$ analỳsiert. Um die Meßergebnisse der Proben unterschiedlicher Aktivität mitteln zu können, wurde die prozentuale Abweichung des einzelnen Meßwertes vom Ausgangswert berechnet.
Die Meßwerte der 10 verschiedenen Seren für den gleichen Zeitpunkt wurden anschließend gemittelt und die zugehörige Standardabweichung berechnet. Wie die Ergebnisse in Abbildung 5 zeigen, ist die Enzymaktivität über einen Zeitraum von 21 Tagen für beide Aufbewahrungstemperaturen stabil. Die Aufbewahrung bei Raumtemperatur zeigte innerhalb von 24 Stunden keinen Aktivitätsabfall.

\section{Präzision}

Zur Bestimmung der Präzision des Verfahrens wurden über einen Zeitraum von 4 Monaten selbst hergestellte Kontrollproben analysiert. Letztere wurden hergestellt, indem aliquote Teile eines gut gemischten Schwangerenpoolserums tiefgefroren und bis zur Analyse bei $-20^{\circ} \mathrm{C}$ aufbewahrt wurden. Die Ergebnisse dieser Untersuchungen sind in Tabelle 1 wiedergegeben.

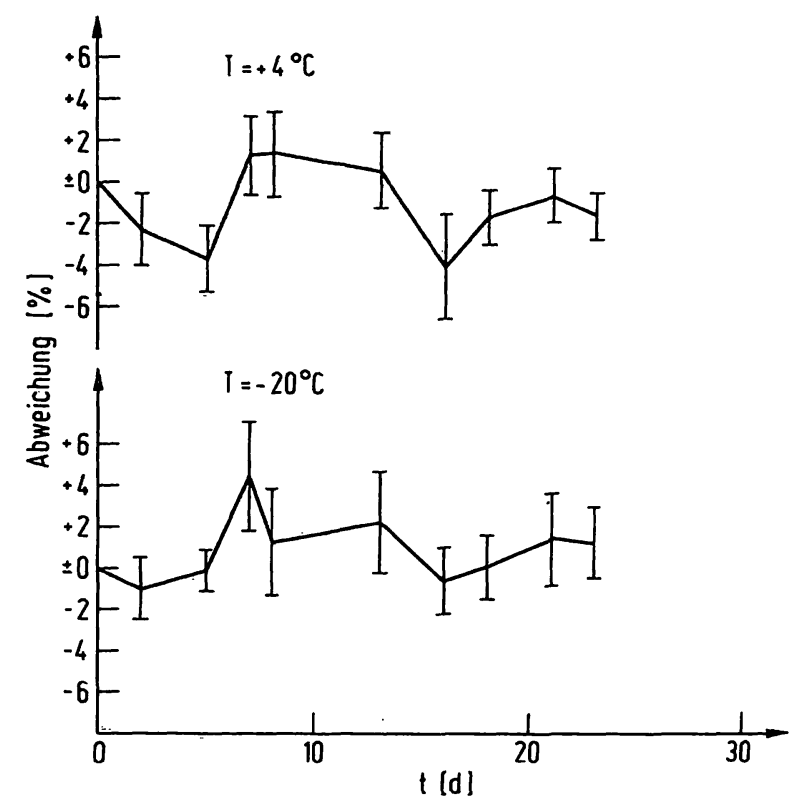

Abb. 5. Mittlere Abweichung der Cystinaminopeptidase-Aktivität von dem Ausgangswert bei unterschiedlichen Aufbewahrungstemperaturen in Abhängigkeit von der Zeit

Tạb. 1. Ergebnisse der statistischen Qualitätskontrolle der Bestimmung der Cystinaminopeptidase-Aktivität mit selbsthergestellten Kontrollproben.

$s_{S}, V_{S}=$ Standardabweichung und Variationskoeffizient in der Serie,

s $_{\mathrm{T}}, \mathrm{V}_{\mathrm{T}}=\operatorname{von} \mathrm{Tag} \mathrm{zu}$ Tag.

\begin{tabular}{lllllll}
\hline Monat & $\begin{array}{l}\bar{x} \\
(U / 1)\end{array}$ & $\begin{array}{l}s_{s} \\
(U / 1)\end{array}$ & $\begin{array}{l}V_{S} \\
(\%)\end{array}$ & $\begin{array}{l}s_{T} \\
(U / 1)\end{array}$ & $\begin{array}{l}V_{T} \\
(\%)\end{array}$ & $n$ \\
\hline Januar & 122 & 3,0 & 2,4 & 4,1 & 3,3 & 4 \\
Februar & 123 & 2,5 & 2,1 & 3,8 & 2,6 & 8 \\
März & 123 & 2,3 & 1,9 & 1,3 & 1,0 & 6 \\
April & 117 & 2,1 & 1,8 & 5,9 & 5,1 & 6 \\
& 121 & 2,5 & 2,1 & 4,1 & 3,4 & 24 \\
\hline
\end{tabular}


Die Streuung in der Serie wurde aus der Differenz von Doppelbestimmungen ermittelt. Die Streuung in einem Lauf des Fast-Analyzers betrug $1,8 \%(\overline{\mathrm{x}}=77 \mathrm{U} / 1$, $s=1,4 \mathrm{U} / 1, \mathrm{n}=15)$. Die Ergebnisse bestätigen die hohe Präzision des Verfahrens. Die Konstanz der Mittelwerte ist ein weiterer Beweis für die Stabilität des Enzyms.

\section{Richtigkeit}

Nach Untersuchungen verschiedener Autoren werden die unterschiedlichen Substrate, die für die Bestimmung der Cystinaminopeptidase-Aktivität benützt werden, von Aminopeptidasen gespalten, die im Serum von Nichtschwangeren vorkommen. Zur Überprüfung dieser Frage wurden 5 verschiedene Seren von Männern analysiert. Die dabei gemessenen Werte betrugen 1-3 U/1. Dieser Versuch zeigt auch, daß Fehler durch Spontanhydrolyse des Substrates vernachlässigbar klein sind. Die Bestimmung der Spontanhydrolyse durch Zugabe von bidest. Wasser anstatt des Serums war nicht möglich, da es dabei durch Ausfällung des Substrates im Reaktionsansatz zu Trübungen kam.

Um einen Hinweis auf die Spezifität der Reaktiọn bzw. eine mögliche unspezifische Erhöhung der gemessenen Cystinaminopeptidase-Aktivität zu bekommen, wurde in Seren von nichtschwangeren Patientinnen mit mechanischem Ikterus, die eine erhöhte Alkalische Phosphatase (EC 3.1.3.1) und Leucinaminopeptidase (EC 3.4.11.1) hatten, die Cystinaminopeptidase-Aktivität bestimmt (Tab. 2).

Tab. 2. Cystinaminopeptidase-Aktivität von nichtschwangeren Patientinnen bei erhöhter Alkalischer Phosphatase und Leucinaminopeptidase.

\begin{tabular}{llll}
\hline Patient & $\begin{array}{l}\text { Alkalische } \\
\text { Phosphatase } \\
\text { (U/l) }\end{array}$ & $\begin{array}{l}\text { Leucinamino- } \\
\text { peptidase } \\
\text { (U/l) }\end{array}$ & $\begin{array}{l}\text { Cystinamino- } \\
\text { peptidase } \\
\text { (U/l) }\end{array}$ \\
\hline 1 & 2550 & 162 & 10 \\
2 & 462 & 54 & 8 \\
3 & 536 & 47 & 13 \\
4 & 637 & 71 & 20 \\
5 & 1405 & 78 & 13 \\
6 & 734 & 86 & 19 \\
7 & 908 & 122 & 14 \\
8 & 1317 & 119 & 9 \\
9 & 370 & 47 & 24 \\
\hline
\end{tabular}

Wie die Ergebnisse zeigen, ist ein Störeinfluß durchaus vorhanden. Allerdings dürfte er am Ende der Schwangerschaft nicht ins Gewicht fallen.

\section{Normalwerte}

Zur Ermittlung vorläufiger Normạlwerte wurden die bisherigen Messungen an schwangeren Patientinnen nach Ausschluß pathologischer Schwangerschaften verwandt (Tab. 3).

Wie die tabellarische Darstellung zeigt, nimmt die Aktivität der Cystinaminopeptidase in den letzten Schwangerschaftswochen zu. Wegen der Größe der biologischen Varianz ist eine transversale Betrachtung für die Beurteilung weniger von Nutzen als die longitudinale Betrachtung. Letztere erfordert wiederholte Bestimmungen in bestimmten Zeitabständen.

Inwieweit die Oxytocinase-Bestimmung als Parameter der foetoplacentaren Einheit von klinischer Relevanz ist, konnte aus den uns zur Verfügung stehenden Daten wegen ihrer häufigen Unvollständigkeit bzw. zu wenigen Kontrollen nicht entschieden werden. Die Literaturangaben sind, wie Kuss (1) in seiner Übersicht ausführt, sehr widersprüchlich. Aus diesem Grunde ist eine prospektive Studie geplant, bei der Human-Plazenta-Lactogen, Cystinaminopeptidase, Oestriol im Serum und die Oestriol-Ausscheidung im Urin bei einer Stichprobe schwangerer Patientinnen ab der 20. Schwangerschaftswoche bestimmt werden sollen.

Tab. 3. Cystinaminopeptidase-Aktivität im Verlauf der letzten Schwangerschaftswochen.

\begin{tabular}{lllr}
\hline $\begin{array}{l}\text { Schwanger- } \\
\text { schaftswoche }\end{array}$ & $\begin{array}{l}\overline{\mathrm{x}} \\
(\mathrm{U} / \mathrm{l})\end{array}$ & $\overline{\mathrm{x}} \pm 2 \mathrm{~s}(\mathrm{U} / \mathrm{l})$ & $\mathrm{n}$ \\
\hline 30 & 59 & $17-101$ & 9 \\
31 & 65 & $33-97$ & 9 \\
32 & 78 & $40-116$ & 10 \\
33 & 87 & $51-123$ & 7 \\
34 & 86 & $38-134$ & 12 \\
35 & 97 & $13-181$ & 16 \\
36 & 107 & $3-211$ & 13 \\
37 & 120 & $22-218$ & 13 \\
38 & 129 & $47-211$ & 19 \\
39 & 139 & $57-221$ & 28 \\
40 & 140 & $40-240$ & 29 \\
\hline
\end{tabular}




\section{Literatur}

1. Kuss, E. (1974), Gynäkologe 7, 124-150.

2. Tuppy, H., Wiesbauer, Ulrike \& Wintersberger, E. (1962), Hoppe Seyler's Z. Physiol. Chem. 329, 278-288.

3. Wintersberger, E., Müller-Hartburg, W. \& Tuppy, H. (1966), Clin. Chim. Acta 14, 786-792.

4. van Oudheusden, A. P. M. (1971), Clin. Chim. Acta 32, 140-141.

5. van Oudheusden, A. P. M. (1972), diese Z. 10, 345-346.

6. Peeters, J. A. B. M. (1972), Clin. Chem. 18, 563-564.
7. Tovey, J. E., Dawson, P. J. G., Fellowes, K. P. \& Fernandez de Castro, A. (1973), Clin. Chem. 19, 756-761.

8. Usategui-Gomez, M., Tarbutton, P. \& Yeager, F. (1973), Clin. Chim. Acta 47, 409-415.

9. Small, C. W. \& Watkins, W. B. (1975), Clin. Biochem. 8, 124-132.

10. Reinouts van Haga, P. (1975), Clin. Chim. Acta 63, 193 bis 195.
Priv. Doz. Dr. Dr. H. Wisser Abteilung für Klinische Chemie Robert-Bosch-Krankenhaus Auerbachstraße 110 D-7000 Stuttgart 50 
